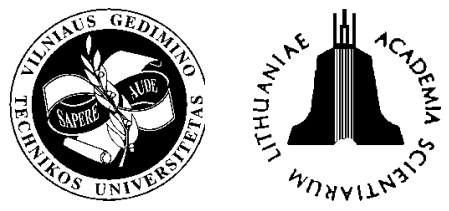

ISSN 1648-4142 TRANSPORT

http:/www.vtu.lt/english/editions

TRANSPORT - 2004, Vol XIX, No 6, 239-251

\title{
THE OPTIMISATION OF LOADING FACILITIES AT THE TERMINAL
}

\author{
Danutė Bagdoniené1 ${ }^{1}$, Skirmantas Mazūra ${ }^{2}$ \\ Dept of Mathematical Statistics, Vilnius Gediminas Technical University, \\ Sauletekio al. 11, LT-10223 Vilnius-40, Lithuania \\ Dept of Transport Management, Vilnius Gediminas Technical University, \\ Plytinès g. 27, LT-10105 Vilnius-40, Lithuania
}

Received 2004-04-02; accepted 2004-10-05

\begin{abstract}
In freight transportation, when a cargo is carried from one terminal to some others (or from several terminals to one area), the delay time of vehicles and loading equipment may be considerable because their operation is not well coordinated. In the presented investigation the appropriate number of transport and loading facilities to ensure their coordinated work at the terminal is determined. The problem of transportation is analysed in the terms of mass service assignment. The problems of stock management and proper choice of transport facilities are defined and analysed taking into account the relationship between the cost of transportation and vehicle capacity and the size of cargo lots. By using mathematical statistical methods, the optimal vehicle capacity is determined for a particular lot on the routes taking the cargo out as well as the periodicity of cargo delivery.
\end{abstract}

Keywords: loading equipment, time of vehicle's turnover, organization of freight transportation.

\section{Introduction}

In the presented paper major functions of enterprises engaged in freight transportation by road which is associated with taking cargoes in and out from the terminal as well as the ways of coordinating the work of loading and transport facilities have been analysed and simulated [1].

The time of harmonized operation of transport and loading facilities may be specified for vehicles which are running or being loaded or uploaded.

The time of transportation depends on the speed and distance covered by a particular transport facility. The speed may be determined when the following factors are taken into account:

- technical parameters of a vehicle including its dynamical characteristics, dimensions, the working order of a vehicle and its engine;

- psychological features of the driver;

- geometrical parameters of the route including physical profile of the downgrade, the area of the traffic lane and road surface;

- general traffic conditions including the type of area (i.e. populated or not populated space), time of

\footnotetext{
1 E-mail: lsaulis@fm.lt

2 E-mail: skirma@centras.lt
}

traffic (i.e. day, night), weather conditions (i.e. rain, snow, fog, etc.), types of transport facilities, traffic intensity, organization and regulation on the road, traffic restrictions, the number of pedestrians, the distance between departure and destination points, and the period of driving without breaks [2].

In transportation factors belonging to various groups have an impact on the vehicle speed.

The conditions associated with the type of road do not vary. Variable conditions include the adhesion of wheels with a road, visibility, mode and intensity of traffic.

Speed may be considered a general criterion of various working conditions of a vehicle determining some operational characteristics [3].

If the speed of a vehicle is assumed to be constant and time losses caused by speed decrease at loading and unloading terminals and the surrounding areas are assumed to be the delay time, the process of scheduling vehicle operation will be considerably simplified [4].

Since the speed of a vehicle depends on many various factors, the impact of which cannot always be precisely evaluated, speed may be considered a random value governed by particular distribution laws.

The solution of transportation organization problems in terms of time is simplified when the value 
sought, which is the speed of a vehicle, is considered an object of statistical observation.

The delay time of a vehicle due to the operations of loading and unloading consists of the transportation processes taking a long time, i.e. loading and unloading, manoeuvring when loading is over, manoeuvring over the area of cargo transfer, lifting sides of a vehicle, fixing the cargo, documentation, etc.

Due to loading and unloading operations the highest standard delay time is determined by the transportation tariffs depending on the methods of cargo loading and unloading as well as on the types of hoisting and loading mechanisms and the shape of cargo [5].

The delay time of a vehicle caused by loading and unloading operations depends on many factors, therefore it may be treated as a random value.

\section{Determining of the demand for transport and load- ing facilities}

The work of transport facilities should be well coordinated with loading/unloading operations.

For any period $T$ of continuous operation including the time interval between the loading (unloading) of the first and the last vehicles a close cooperation is needed. This implies that

$$
\frac{\sum_{i=1}^{n} t_{a p i}}{A_{e}}=\frac{\sum_{i=1}^{n} t_{k i}^{\prime}}{N_{k}},
$$

here $t_{a p i}$ is the time of $i$-th vehicle turnover; $n-$ number of vehicle turnovers in time, $T ; N_{k}$-number of loading machines; $A_{e}$ - number of operating vehicles; $t_{k i}^{\prime}$ - time of loading $i$-th vehicle including the time of waiting for its arrival.

If the average time of vehicle turnover is as follows:

$$
\bar{t}_{a p}=\frac{\sum_{i=1}^{n} t_{a p i}}{n},
$$

while the average loading time and time of waiting for the vehicle to arrive may be written in this way:

$$
\overrightarrow{t_{k}}=\frac{\sum_{i=1}^{n} t_{k i}^{\prime}}{n},
$$

then, the operation of vehicles and loading/unloading devices will be coordinated if:

$$
\frac{\bar{t}_{a p}}{A_{e}}=\frac{\vec{t}_{k}}{N_{k}},
$$

The average time of vehicle turnover is expressed as:

$$
\bar{t}_{a p}=\bar{t}_{g r}+\bar{t}_{b}+\bar{t}_{\text {apt }},
$$

here $\bar{t}_{g r}$ is an average time interval between the moment when the handling of a vehicle at the loading area is over up to its coming back to the same terminal (vehicle's return time); $\bar{t}_{b}-$ idle time of the vehicle waiting for loading/unloading; $\bar{t}_{\text {apt }}-$ time of vehicle handling (loading/unloading).

The average loading time and time of waiting for vehicle arrival:

$$
\vec{t}_{k}=\bar{t}_{a p t}+\vec{t}_{b},
$$

here $\overrightarrow{t_{k}}$ - average delay time of loading devices due to waiting for the vehicle to arrive.

The time $\bar{t}_{a p}$ and $\overrightarrow{t_{k}}$ depend on $\bar{t}_{b}$ and $\vec{t}_{b}$, i.e. on the number of the available transport and loading facilities as well.

If we assume that:

$$
\vec{t}_{k}=\bar{t}_{k}=\bar{t}_{a p t}+\bar{t}_{b},
$$

then, the condition (4) will be of the form:

$$
\frac{\bar{t}_{a p}}{A_{e}}=\frac{\bar{t}_{k}}{N_{k}} .
$$

Let the time $\bar{t}_{g r}$ referring to vehicle return to the initial point does not depend on the number of vehicles, then, taking into account the condition (4), we may write the following expression:

$$
\frac{\bar{t}_{g r}+\bar{t}_{a p t}}{A_{e}} \varepsilon=\frac{\bar{t}_{a p t}}{N_{k}},
$$

here $\varepsilon$ is a coefficient expressing the coordination of operation of transport and loading facilities.

The value of the coefficient $\varepsilon$ in calculations should correspond to the optimal relationship between the delay time of transport and loading facilities. Usually, the optimal value is $\varepsilon<1$, however, in some cases, $\varepsilon>1$ is used to ensure minimal delay time of loading equipment. For the harmonized work, we get $\bar{t}_{b}=\vec{t}_{b}=0$ and $\varepsilon=1$ by eliminating delay time caused by other reasons.

The work of transport and loading equipment at the terminals may be coordinated by choosing the required number of vehicles.

If the calculated number of vehicles needed to coordinate the work with the available loading equipment at the loading terminal is fewer than the total number of vehicles set aside for this terminal from all unloading areas, then either the latter number should be decreased or the capacity of the loading terminal increased so that the following conditions are satisfied: 


$$
A_{p i} \geq \sum_{j=1}^{n} A_{i s i j}, i=1,2, \ldots, m,
$$

here $A_{p i}$ the calculated number of vehicles for $i$-th loading terminal; $A_{i s ̌ i j}$ - number of vehicles loaded in $i$-th and unloaded in $j$-th terminal. minal.

The same applies to any loading/unloading ter-

Organizing transportation in this way special attention should be paid to the proportion of truck tractors and trailers ensuring well-coordinated work of transport and loading equipment. Similar problems should be solved when removable truck bodies and multiusage containers are employed.

Various schemes of a 'shooting' method exist inclu-ding the coupling of trailers only in one of 'pendulum' (half-shooting) route points. Given $n$ points on a route of bringing the cargo in and out by one truck, the number of trailers should not be lower than $(n+1)$, with one trailer for working with a truck and others kept at the terminals.

General conditions of the operation of trucks and trailers may be expressed as follows:

with respect to loading/unloading terminals:

$$
\frac{\bar{t}_{a p}}{A_{V . e}}=\frac{\bar{t}_{k}+\bar{t}_{p . p}}{P_{k}},
$$

for loading operations:

$$
\frac{\bar{t}_{a p}}{A_{V . e}}=\frac{\bar{t}_{p a k}+\bar{t}_{p . p}}{P_{p a k}}
$$

for unloading operations:

$$
\frac{\bar{t}_{a p}}{A_{V . e}}=\frac{\bar{t}_{i \check{s}}+\bar{t}_{p . p}}{P_{i \check{s}}},
$$

here $A_{V . e}$ - number of trucks, $\bar{t}_{a p}$ - average time of truck turnover;

$$
\bar{t}_{a p}=\frac{l_{m}}{v_{T}}+\bar{t}_{p \cdot p} n=\frac{l_{m}+v_{T} \bar{t}_{p \cdot p} n}{v_{T}},
$$

here $l_{m}$ - route length (distance covered per turnover), $\mathrm{km} ; \bar{t}_{p . p}$ - average time of trailer's recoupling, h.; $n$ - number of terminals for bringing the cargo in and out on the route; $\bar{t}_{k}, \bar{t}_{p a k}, \bar{t}_{i \check{s}}$ average time of loading, loading up and unloading a semi-trailer (including the delay time due to performing these operations); $v_{T}$-speed of a vehicle; $P_{k}, P_{p a k}, P_{i \check{s}}-$ a number of semi-trailers available at loading, loading up and unloading areas, respectively:

$$
P_{k}=\frac{\bar{t}_{k}+\bar{t}_{p \cdot p}}{\bar{t}_{a p}} A_{V . e},
$$

$$
\begin{gathered}
P_{p a k}=\frac{\bar{t}_{p a k}+\bar{t}_{p \cdot p}}{\bar{t}_{a p}} A_{V . e}, \\
P_{i \check{s}}=\frac{\bar{t}_{i \check{s}}+\bar{t}_{p \cdot p}}{\bar{t}_{a p}} A_{V . e},
\end{gathered}
$$

If a vehicle has only one semi-trailer, the total number of semi-trailers (engaged in transportation and loading and unloading operations) will be as follows:

$$
P=A_{V . e}+P_{k} n_{k}+P_{p a k} n_{p a k}+P_{i \check{s}} n_{i \check{s}},
$$

here $n_{k}, n_{p a k}, n_{i \check{s}}$ are areas of loading, loading up and unloading operations on the route

By substituting the values of $P_{k}, P_{p a k}, P_{i \check{s}}$ into the formula (17) and taking into account that $n_{k}+n_{\text {pak }}+n_{i \check{s}}=n$, we will obtain:

$$
P=A_{V . e}\left[1+\frac{1}{\bar{t}_{a p}}\left(\bar{t}_{k} n_{k}+\bar{t}_{p a k} n_{p a k}+\bar{t}_{i \check{s}} n_{i \check{s}}+\bar{t}_{p \cdot p} n\right)\right],
$$

By using the formula (18), the number of semitrailers needed for any type of route may be calculated.

The number of semi-trailers for a vehicle (truck) is as follows:

$$
m_{a}=\frac{P}{A_{V . e}} .
$$

In the case of a pendulum route, when a loaded semi-trailer is delivered directly to a destination point and back $\left(l_{m}=2 l_{\text {pak.v }}, l_{\text {pak.v }}\right.$ is a distance covered by a loaded vehicle per run, $\mathrm{km} ; n=n_{k}=2 ; n_{p a k}=n_{i \check{s}}=0$ ) we have:

$$
m_{a}=1+\frac{\left(\bar{t}_{k}+\bar{t}_{p . p}\right) v_{T}}{\bar{l}_{p a k . v}+v_{T} \bar{t}_{p . p}}
$$

For the case of a pendulum route with an empty vehicle on the way back $\left(l_{m}=2 l_{\text {pak.v }} ; n=2\right.$; $\left.n_{\text {pak }}=n_{i \check{s}}=1 ; n_{k}=0 ; t_{p a k}+t_{i \check{s}}=t_{k}\right)$, we get:

$$
m_{a}=1+\frac{\left(\bar{t}_{k}+2 \bar{t}_{p \cdot p}\right) v_{T}}{2\left(\bar{l}_{\text {pak.v }}+v_{T} \bar{t}_{p . p}\right)} \text {. }
$$

In the case of a circular triangle route $\left(l_{m}=\frac{2 \bar{l}_{p a k . v}}{\beta}\right.$, where $\bar{l}_{p a k . v}$ is an average distance covered by a loaded vehicle per run; $\beta$ is the vehicle's usage coefficient; $\left.n=3 ; n_{k}=n_{p a k}=n_{i \check{s}}=1 ; \bar{t}_{i \check{s}}+\bar{t}_{p a k}=\bar{t}_{k}\right)$ we obtain:

$$
m_{a}=1+\frac{\left(2 \bar{t}_{k}+3 \bar{t}_{p . p}\right) v_{T} \beta}{2 \bar{l}_{p a k . v}+3 v_{T} \beta \bar{t}_{p . p}}
$$


If a truck has more than one trailer (semi-trailer), the need for them is proportionally higher. As one can see from the above relationships, the number of semi-trailers needed is higher if their delay time for loading and unloading $\bar{t}_{i s}, \bar{t}_{p a k}$ is longer, the speed of a vehicle $v_{T}$ is higher and the distance of transportation $\bar{l}_{p a k . v}$ is shorter. Taking this into account we may state that the number of semi-trailers is directly proportional to the time $\bar{t}_{p a k}, \bar{t}_{i \check{s}}$, while its relationship with $v_{T}$ and $\bar{l}_{p a k . v}$ is hyperbolic. For large distances $m_{a}$ is approaching 1 , when $l_{p a k . v} \rightarrow \infty$, i.e. $\lim _{l_{p a k . v \rightarrow \infty}} m_{a}=1$.

\section{Scheduling of the operation of transport facilities}

Traffic scheduling aims to minimize the delay time of vehicles and loading equipment when their work is not coordinated. Solving the problem will let us to optimize the schedules of vehicle operation and coordinate the work of particular transport facilities at the terminals.

Regular arrival and departure of vehicles at loading and unloading terminals primarily depend on a rhythmic pace of their work. A schedule should ensure regular arrival of vehicles for the initial loading, coordinate breaks and vehicle arrival when the breaks are over.

When vehicles do not arrive in time, this may increase the delay time of vehicles and loading equipment in transporting the cargo from one area to the other (or vice versa). Let us consider a solution of this problem providing for the coordination of work of rolling stocks and loading/unloading equipment when the cargoes are transported from one area to many others.

Two common cases are possible. Let the vehicles operate on the route with the same freight delivery distances and be empty on their way back. The required number of vehicles will be as follows:

$$
A_{e}=\frac{t_{a p}}{t_{p a k}},
$$

here $t_{a p}$ - time of vehicle turnover or the total time of loading and travel to the point of destination and back; $t_{p a k}$ - time of loading a vehicle.

If the turnover values $t_{a p}$ and $t_{p a k}$ are the same, the number of vehicles obtained from the formula (23) may ensure the continuous work of loading equipment and help to avoid the delays associated with loading.

Generally the solution of the problem becomes more complicated.

Let one loading mechanism be available at the loading area and vehicles of the same capacity $q$ be used for transportation. Vehicles will deliver the cargo directly to customers $B_{1}, B_{2}, \ldots, B_{j}, \ldots, B_{n}$ (or to their groups) in the amounts $P_{1}, P_{2}, \ldots, P_{j}, \ldots, P_{n}$. Thus, $n_{r j}=\frac{P_{j}}{q \gamma_{s t}}$ hauls will be made delivering the cargo to every customer $B_{j}$ or one haul to every $j$ th group of customers involved in the dispatching route $\left(\gamma_{s t}\right.$ is coefficient of static use of vehicle capacity) will be made. When the cargo is delivered, the vehicle returns to the initial point. The time of vehicle turnover on the route $j$ is equal to $t_{a p j}$ and the time of loading is $t_{p a k j}=t_{p a k}$.

The work may be organized in the following way:

- loading equipment should work without breaks during the shift, if the scheduling of transportation is to be observed;

- some delay time of loading equipment is allowable when vehicles are being loaded.

In both cases the minimum required number of vehicles to deliver the cargo should be determined. At the same time the sequence of the departure of vehicles should be established to minimize time losses by vehicles in the first case or the total time losses in the second.

\section{Analysis of work coordination of transport and loading facilities taking account random factors}

The speed and delay time of vehicles (in loading and unloading) depend on many factors. Therefore, time losses in transportation should be considered as a random value. In fact, traffic does not always keep to the schedule based on the average speed and delay time of vehicles. This means that it is important to provide for possible deviations and analyse their reasons.

Time of cargo delivery on the vehicle departure routes depends on the type of route, speed and delay time of the vehicle at the loading area and at any intermediate point. The sequence of points is determined not only by the total distance run by a vehicle, but also by the time of its arrival at each point.

Specifying the speed of a vehicle on the departure route the fact is that the distances between intermediate points are usually short, not exceeding 2 $3 \mathrm{~km}$. To simplify scheduling the speed of a vehicle is assumed to be uniform and time losses at loading areas are considered to be the delay time.

In assessing the total costs, the delay time is considered to increase when the number of deliveries grows.

In delivering very small lots of cargo, the time for technological operations and that spent for preparation/completion of operations at any intermediate point should not be calculated separately because it usually exceeds the scheduled time. It is better first to 
determine the total time of unloading $\left(t_{i \check{s}}\right)$ at any intermediate point and then to find the time of preparation/completion of operations. This would allow us to express the relationship between the above time and the size of cargo lot $\left(g_{d}\right)$ in the following way:

$$
t_{i \check{s}}=t_{t} g_{d}+t_{u \check{z}},
$$

here $t_{t}$ - time of technological operations relating to unloading a unit cargo mass; $t_{u \check{z}}$ - additional time of driving in the intermediate terminal.

In Fig 1 statistical relationship between the delay time of tank vehicles at the intermediate points of freight transportation routes and the size of cargo lot is presented. The delay time of a vehicle at any point depends on some random factors. This accounts for the various total time of performing the work in particular cases. It is important to determine the allowable deviations ensuring the arrival of a particular vehicle at the specified points.

If the number of hauls is small, time losses, i.e. the delay time of a vehicle may be planned according to its maximum value. However, the larger the number of hauls, and especially when it exceeds 10 , the more the above assumption deviates from the expected time of arrival, because in this case the unfavourable conditions can hardly occur again.

A measure of random value variation may be considered its mean square deviation. It may be stated that possible time of transportation ranges from $-\Delta t=k_{1} \sigma_{T}$ to $+\Delta t=k_{2} \sigma_{T}$ (here $k_{1}$ and $k_{2}$ are coefficients to be applied depending on the determined time expenditures; $\sigma_{T}$ is average square time $T$ deviation in transportation).

On the dispatching routes with a large number of intermediate points for vehicle driving in, speed variation may be neglected because of the influence

$t_{i \check{s}}, \min$

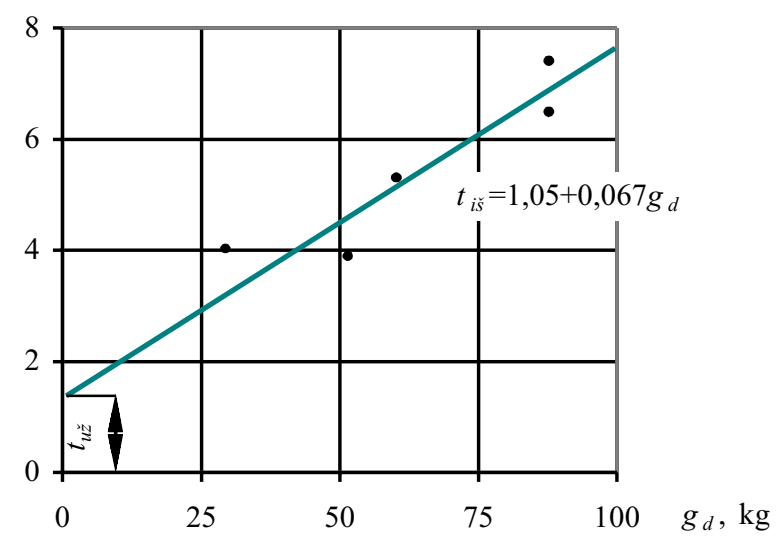

Fig 1. The relation between vehicle delay time at loading terminals and the lot size of cargo weight on the delay time of vehicles and its deviation from the average value. Then, for delivering cargoes to the point $n$, the following expression is valid:

$$
\sigma_{T}^{2}=\sum_{i=1}^{n} \sigma_{T i}^{2}=\frac{n \sum_{j=1}^{m}\left(t_{p j}-\bar{t}_{p}\right)^{2}}{m},
$$

here $\bar{t}_{p}$ is average vehicle delay time at intermediate points, min; $t_{p j}$ is the delay time at intermediate points for $j$-th observation, min; $m$ - number of observations.

In predicting the time of transportation on various routes random value $t_{p j}$ should be considered with respect to two random factors: organizational conditions at the intermediate points and the amount of cargo $\left(g_{d}\right)$ delivered to each point, where the limitations are applied only to $q \gamma_{s t}=\sum_{i=1}^{n_{u \breve{z}}} g_{d i}$ and the number of drivings in $n_{u \check{z}}$ is known.

Scheduling traffic and loading equipment operation the evaluation of the impact of random factors in transportation allows the losses to be considerably reduced.

\section{Organization of the continuous work of transport and loading facilities as a problem of mass service}

The operational characteristics of mass service may be determined based on various criteria. Major of them are the total costs of loading/unloading one ton of cargo or of one vehicle loading/unloading operation and relative operation efficiency in a system (at a loading/ unloading terminal). For the sake of simplicity of calculation only the costs relating to losses as part of the total costs characterizing the efficiency of operation may be considered.

The total losses of the system per hour may be obtained from the following expression:

$$
\sum S_{v a l}=C_{p r}\left(\bar{r}+\bar{n}_{u \check{z}}\right)+C_{p r}^{\prime} \bar{n}_{o}+C_{d a r}^{\prime} \bar{n}_{u \check{z}},
$$

here $C_{p r}$ - losses due to one hour delay of a vehicle, $\mathrm{Lt}$; $C_{p r}^{\prime}-$ losses due to one hour delay of a loading/ unloading mechanism, Lt; $C_{d a r}^{\prime}$ - cost of one hour operation of a loading/unloading mechanism, cond. units, $\bar{r}$ - average number of transport facilities used.

Cost value of one hour delay of transport and loading/unloading facilities in a system for other reasons is:

$$
\Delta S_{v a l}=C_{p r} \bar{r}+C_{p r}^{\prime} \bar{n}_{o} .
$$

The total costs and the value of losses calculated for one vehicle are as follows: 


$$
\sum S_{k}=\frac{\sum S_{v a l}}{n}\left(\vec{t}_{b}+\bar{t}_{a p t}\right),
$$

or

$$
\Delta S_{k}=\frac{\Delta S_{v a l}}{n}\left(\bar{t}_{b}+\bar{t}_{a p t}\right)
$$

By making transformations and taking into account (26) and (27) we get:

$$
\begin{gathered}
\Delta S_{k}=\frac{\bar{t}_{a p t}}{\bar{n}_{u} \cdot}\left(C_{p r} \bar{r}+C_{p r}^{\prime} \bar{n}_{o}\right)=C_{p r} \bar{t}_{b}+C_{p r}^{\prime} t_{b}^{\prime}, \\
\sum S_{k}=\bar{t}_{a p t}\left[C_{p r}\left(\frac{\bar{r}}{\bar{n}_{u \check{z}}}+1\right)+C_{p r}^{\prime} \frac{\bar{n}_{o}}{\bar{n}_{u \check{z}}}+C_{p r}^{\prime}\right] .
\end{gathered}
$$

It follows from (29) and (30) that

$$
\sum S_{k} \check{z} \Delta S_{k}+\left(C_{p r}+C_{d a r}^{\prime}\right) \bar{t}_{a p t} .
$$

Since the total costs $\left(C_{p r}+C_{d a r}^{\prime}\right) \bar{t}_{a p t}$ do not depend on the controlled parameters, the optimal criteria of the system functioning (30) and (31) are equally important. When solving the optimization problem based on minimal costs of the output unit (1t), the relationship (31) should be used.

The delay losses experienced for other reasons calculated for one loading operation $\Delta S_{k}$ are related to losses of one hour in a loading operation of a vehicle, that is:

$$
\Delta S_{k}=\frac{\Delta S_{v a l}}{n}\left(\bar{t}_{b}+\bar{t}_{a p t}\right)=\Delta S_{v a l} \frac{\bar{t}_{a p t}}{\bar{n}_{u \check{z}}} .
$$

The second optimization criterion ( $\Delta S_{k} \rightarrow \min$ ) corresponding to the requirements of transportation organization based on minimum cost of the output unit is more appropriate. The evaluation of total costs variation when the costs changed with respect to the work for an hour $\Delta S_{v a l}$ contains a slight error, as shown by the equation (33), not only for the case when $n_{u \check{z}} \rightarrow n$, or $\omega_{k}=\left(1-\frac{n_{u \check{z}}}{n}\right) \rightarrow 0$.

The labour input in cargo loading/unloading is expressed in this way:

$$
\sum I_{k}=\left(\bar{r}+\bar{n}_{u \check{z}}+n R_{k}\right) \frac{\bar{t}_{b}+\bar{t}_{a p t}}{n}=\bar{t}_{a p t}\left[\frac{1}{\bar{n}_{u \check{z}}}\left(\bar{r}+n R_{k}+1\right)\right],(34)
$$

here $R_{k}$ is a number of workers engaged in loading operations or working with loading equipment.

It is advisable that a driver of a vehicle be around waiting for loading operations to be over.
Non-technological idle time of drivers and loaders during the loading of one vehicle is expressed as follows:

$\Delta I_{k}=\left(\bar{r}+\bar{n}_{o} R_{k}\right) \frac{\vec{t}_{b}+\bar{t}_{a p t}}{n}=\frac{\bar{t}_{a p t}}{\bar{n}_{u \check{z}}}\left(\bar{r}+\bar{n}_{o} R_{k}\right)=\bar{t}_{b}+\vec{t}_{b} R_{k}$

It is clear that labour input into minimal total and relative operations in the loading area depends on the choice of control parameters when time losses are minimal. The losses are found from the relationship (35) to be used in solving the problem of optimal system operation based on the criterion of minimal labour input.

Analytical models of the theory of mass service are aimed to satisfy the requirements of stationary Poisson's flow and exponential service time. However, in many cases major characteristics of loading areas do not meet these conditions. The requirements raised for services are not always constant. Three phases of operation of loading terminals may be distinguished including the initial, stable mode and final stages.

The unstable operation can be observed before and after the breaks.

Poisson's and exponential distribution describes random uncontrolled actions. If they may be applied to describe the operation of loading/unloading terminals, this signals about poor organization of transportation and loading processes which need to be considerably improved.

However, analytical models based on Poisson's flow and exponential service time distribution are the simplest analogues of loading terminal functioning. By their analysis one can get the insight into the nature of the above processes. In some cases the results of the decisions made by using these models may be considered as boundary conditions.

Let us consider a simple closed system of mass service (Fig 2). There are $m$ vehicles (demands) serviced by $n$ loading mechanisms. Every vehicle makes a flow of demands $\lambda=\frac{1}{\bar{t}_{g r}}$, though when it is not ser-

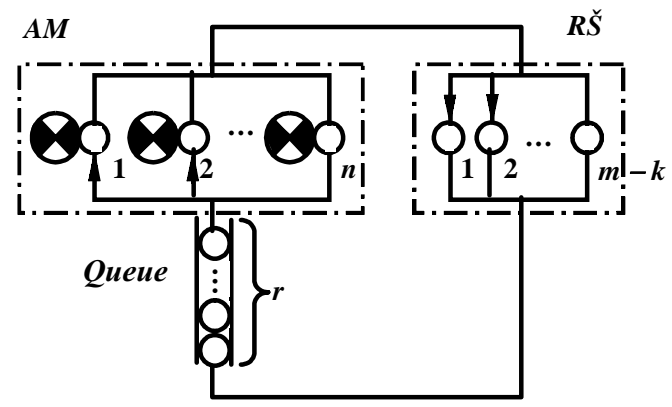

Fig 2. An operational scheme of a mass service closed system: $A M$ - service unit, $R \check{S}$ - source of demand 
viced, the flow of demands ends in the time of servicing. The time of satisfying (servicing) one demand makes $\bar{t}_{a p t}=\frac{1}{\mu}$. If at the time of vehicle arrival there are no free loading mechanisms, it should wait in a queue for an unlimited period of time for servicing.

In a discrete system with continuous waiting time, the pace of vehicle arrival at the loading area is proportional to the number of vehicles outside the above area, while, if there are no vehicles at the loading area, it is equal to $m \lambda$. The higher the number of vehicles in a queue, the lower the intensity of the flow, and vice versa.

One of the major characteristics of mass service is $\xi$, representing the probability that a vehicle can be outside the loading area at any moment of time. The value $\xi$ is also referred to as a coefficient of the efficient use of equipment (a vehicle in this case). It may be calculated from the formula:

$$
\xi=1-\frac{\bar{k}}{m},
$$

here $\bar{k}=\bar{n}_{u \check{z}}+\bar{r}$ is an average number of vehicles at the loading area.

The probability that a vehicle will be outside the loading area $\xi$ may also be determined from the formula:

$$
\xi=\frac{\bar{t}_{g r}}{\bar{t}_{g r}+\bar{t}_{k}},
$$

here $\bar{t}_{g r}=\frac{1}{\lambda}$ is the average time of the vehicle's return, while the average time of loading the vehicle at the loading area is as follows:

$$
\bar{t}_{k}=\bar{t}_{g r} \frac{1-\xi}{\xi} .
$$

The above time consists of the time of waiting in a queue and the time of service. The average time of servicing a vehicle is $\bar{t}_{a p t}=\frac{1}{\mu}$. It follows that the average waiting time (in a queue) may be described as:

$$
\bar{t}_{b}=\bar{t}_{k}-\bar{t}_{a p t}=\frac{1}{\lambda} \cdot \frac{1-\xi}{\xi}-\frac{1}{\mu} .
$$

\section{Simulating of the work of transport and loading facilities}

Let the system of mass service consist of stages at which several pieces of service equipment operate. If all the facilities available at a particular stage are working, then the needs (demands) which were satisfied (serviced) at an earlier stage, are waiting in a queue. This system consists of several stages of service. At each stage a queue is formed. If, after being serviced at $N$-th stage, a vehicle returns back to the initial stage, such a system is referred to as a closed system.

Mass service systems including multilinear and multistage systems as well as their connections with other systems, are referred to as a mass service network. A process of transportation may be described in terms of mass service network performance, the service stages of which are assumed to be a sequence of loading/unloading operations as well as the movement of a loaded or empty vehicle on particular route sections.

The operation on a particular route with a servicing sequence is similar to a transporting conveyor. If we imagine this process as a mass service network, the appropriate conclusions may be made.

The loading of a vehicle is carried out at $i$-th point, its duration is expressed by a random value. In order to construct an analytical model let us assume that the value is distributed according to the exponential law with the parameter $\mu_{i}=\frac{1}{\bar{t}_{\text {apti }}}$.

The distance run by a vehicle from $i$-th to $(i+1)$ th point is a constant value. It corresponds to the constant time of the vehicle movement $\tau_{i}$.

Let us analyse the solution of this problem as the work of a conveyor system which may be considered equivalent to the operation of vehicles on particular closed routes.

Each point (road section) with the stable servicing sequence $\tau_{i}$ may be substituted by imaginary points $z$, at which the time of service duration is distributed exponentially with the parameter $\frac{z}{\tau_{i}}$, $(i=1,2, . ., n)$. If the vehicles at imaginary points are not delayed by waiting for servicing, then the average total time and the average square deviation are equal to $\tau_{i}$ and $\frac{\tau_{i}}{z}$, respectively (if $z$ approaches a constant value). Thus a service system with imaginary points may be used as good approximation analysing the operation of vehicles on separate routes.

Providing the real and imaginary service points with numbers according to their order of priority depending on the results obtained for closed systems we may consider that there are $m_{s}$ vehicles at $s$-th point $(s=1,2, \ldots, N)$. Even when all $m$ vehicles would be found on some road section connecting two points (imaginary points of $i$-th group), a probability that all vehicle $m$ will be at one of the imaginary points 
approaches to zero when $z$ is increasing. If at a particular route section a smaller number of vehicles $m$ is found, then a probability that the queues will be formed at the imaginary points approaches to zero proportionally to the number of points. In a boundary case when $z \rightarrow \infty(N \rightarrow \infty)$, a probability that $m_{i}$ vehicles are at $i$-th point, with the independent distribution of vehicles at the route section, may be found as follows:

$$
\begin{aligned}
& p\left(m_{1}, \ldots, m_{n}\right)=\frac{m !}{\left(m-\sum_{i} m_{i}\right)} v_{1}^{-m_{1}} \ldots v_{n}^{-m_{n}} p(0, \ldots, 0), \\
& p(0, \ldots, 0)=\left[\int_{0}^{\infty} \ldots \int_{0}^{\infty}\left(\sum_{i=1}^{n} x_{i}+1\right)^{m} \prod_{i} v_{i} e^{-\sum_{i=1}^{n} v_{i} x_{i}} d x_{1}, \ldots, d x_{n}\right]^{-1},
\end{aligned}
$$

implying that there are no vehicles at all loading areas;

$$
\begin{aligned}
& v_{i}=\mu_{i} \tau \\
& \tau=\sum_{i=1}^{n} \tau_{i} \text { is time of vehicle's turnover. }
\end{aligned}
$$

The equation (40) determines the distribution of vehicles at particular points and road sections on particular closed circular routes including random loading areas.

\section{Statistical modelling of the operation of transport and loading facilities}

To solve the problems of organizing the work in the loading areas, time characteristics of the observed mass servicing can not be always used for determining the major parameters of the analytical approach relating to the performance of mass service systems theory.

The variation of the above system under various operational conditions may be determined by constructing a model based on the collected statistical data.

Let us consider its application to the joint operation of vehicles and excavators.

Since vehicles arrive for servicing (loading) randomly, and the duration of the process and the return time of a vehicle are also random values, then at the particular time both vehicles and excavators may be idle. Depending on the number of vehicles, the delay time and the cost of the vehicle output and transportation of 1 ton $\left(1 \mathrm{~m}^{3}\right)$ of earth may vary.

The data obtained describe the results of special analysis of random time values referring to the return of the vehicle $T_{g r}$ and its handling $T_{a p t}$ (if they are normally distributed, it is sufficient to have their average values $\bar{t}_{g r}, \bar{t}_{a p t}$ and the dispersion $\sigma_{t_{g r}}^{2}, \sigma_{t_{a p t}}^{2}$, the minimum and the maximum number of vehicles $m_{\min }, m_{\max }$, as well as $n_{e}$ simulations of the demands for servicing ensuring precise calculation, i.e. the sample determined by commonly used statistical methods):

To determine the above volume $n$, the average random value $t$ should not differ from the mathematical $m_{t}$ by more than $\varepsilon$ with a given probability:

$\beta_{p a t}=P\left(\left|\bar{t}-m_{t}\right|<\varepsilon\right)$.

A relative error for a given sample is as follows:

$$
\delta=\frac{\varepsilon}{t}=\frac{t_{\beta_{p a t}} \cdot S}{\sqrt{n} \bar{t}},
$$

here $t_{\beta_{p a t}}$ - confidence of a given probability of the obtained result $\beta_{p a t} ; S$-mean square cost error; $\varepsilon$ - coefficient expressing the coordination degree of operation of transport and loading facilities.

The relationship $\frac{S}{\bar{t}}$ may be obtained by evaluating a sample of 15-20 pieces of data.

Based on the expression (43) the graphs $n=f\left(t_{\beta_{p a t}}, \delta, \frac{\bar{S}}{\bar{t}}\right)($ Fig 3$)$ for the case $\beta_{p a t}=0,95$, $t_{\beta_{\text {pat }}}=1,96$ were made.

A given system is closed. A flow of demands (vehicles) is formed when the individual flow of demands for each vehicle is formed. Its intensity is a variable which is equal to zero when all vehicles are waiting in a queue and in the loading area. However, when there are no vehicles in the loading area it reaches the maximum value.

Let us consider the modelling of $i$-th vehicle (demand) arrival and handling (Fig 4). In this case the data obtained in modelling the previous $(i-1)$ th demand handling should be partially known: $t_{i-1}^{p a b}-$ time of completing the previous $(i-1)$-th demand

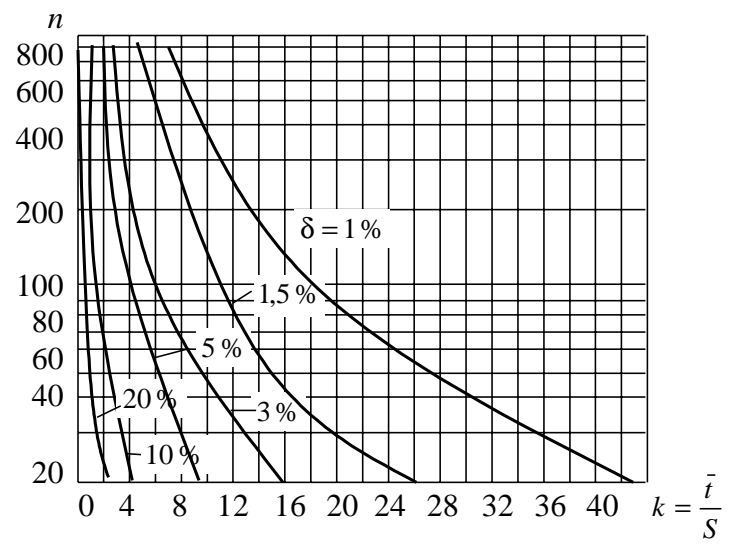

Fig 3. Graphical representation of determining the required sample 


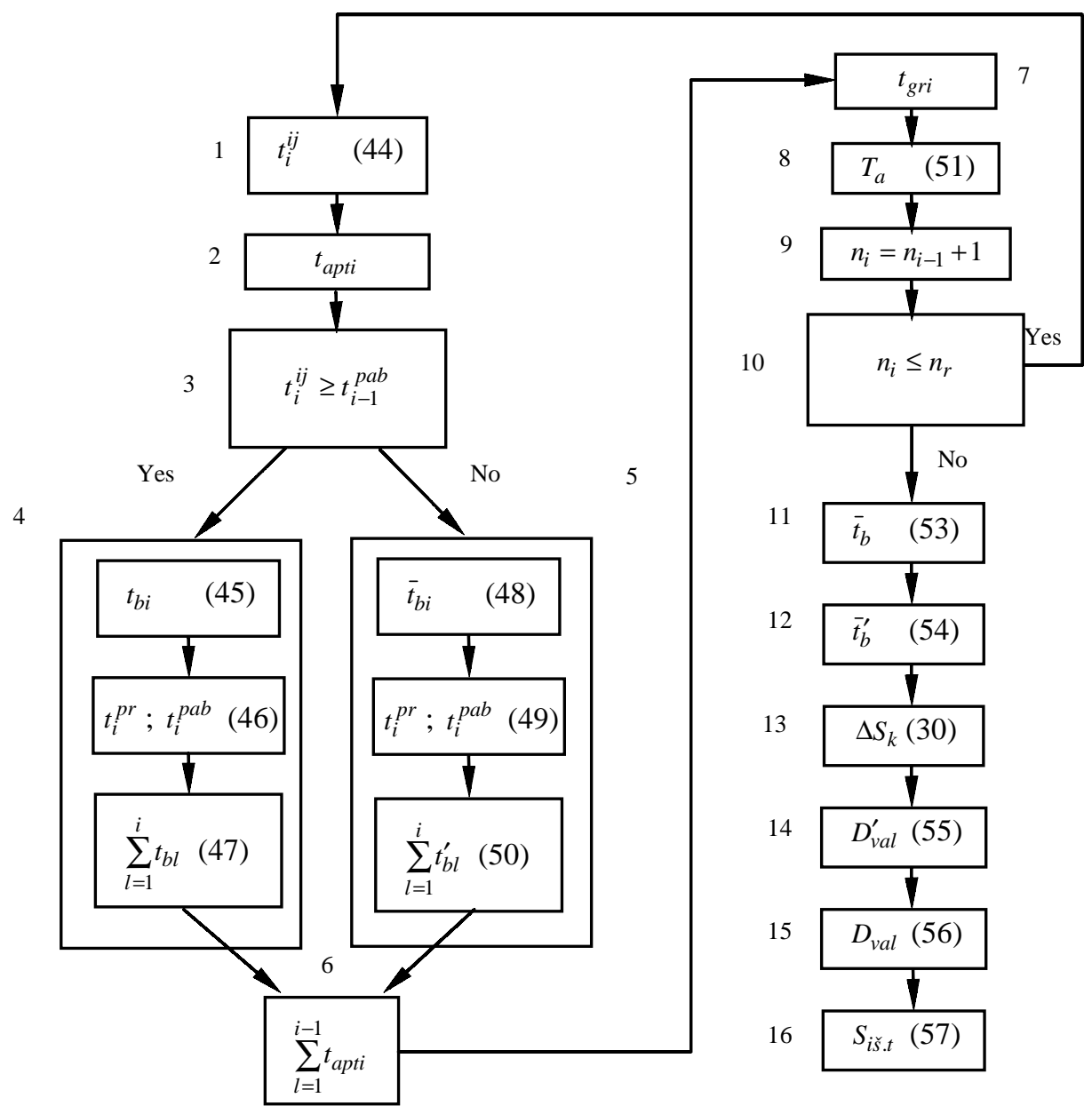

Fig 4. A diagram representing the simulation of the joint work of vehicles and excavators

handling; $T_{k}(k=1,2, \ldots, m)$ - all moments of the $k$-th vehicle arrival and loading; $\sum_{l=1}^{i-1} t_{\text {aptl }}$ - the total service time of the previous demands (from 1 to $(i-1)$ ); $\sum_{l=1}^{i-1} t_{b l}$ - the total delay time of vehicles (demands) waiting for handling and of excavators waiting for demands; $n_{i-1}$ - number of demands handled till $i$-th demand was obtained.

In modelling the first demand the above values except for the moment $T_{k}$ formed according to the specified distribution laws, are equal to zero.

The iteration 1 was used to determine the moment of the arrival of $i$-st vehicle (demand) at the system $t_{i}^{i j}$. This will be denoted by $a$ :

$$
t_{i}^{i j}=\min T_{k}=T_{a}
$$

here $T_{k}$-moments of the arrival of different vehicles, $k=1,2, \ldots, m$.

If there are several equal minimal $T_{k}$ values, a vehicle with the smallest number in the queue is handled.
The iteration 2 is used to obtain the time of handling $i$-th demand (loading of vehicle) $t_{\text {apti }}$ according to the given distribution laws.

The iteration 3 compares the moments of the arrival of $i$-th demand $t_{i}^{i j}$ and the completion of $(i-1)$ th demand $t_{i-1}^{p a b}$ by excavator. If $t_{i}^{i j} \geq t_{i-1}^{p a b}$, the excavator is free and the transfer to the 4-th step is possible; in the opposite case, the 5-th iteration is used.

With the help of the iteration 4 the delay time of $i$-th demand (vehicle) $t_{b i}$ due to waiting for handling is determined:

$$
t_{b i}=t_{i}^{i j}-t_{i-1}^{p a b} .
$$

Then, the initial and the final time of $i$-th demand handling is determined:

$$
\left.\begin{array}{c}
t_{i}^{p r}=t_{i-1}^{p a b}, \\
t_{i}^{p a b}=t_{i}^{p r}+t_{a p t i},
\end{array}\right\}
$$

and the total waiting time of all previously arrived $l$ demands, also including the delay time of vehicles (demands) before servicing is as follows: 


$$
\sum_{l=1}^{i} t_{b l}=\sum_{l=1}^{i-1} t_{b l}+t_{b i}
$$

The iteration 5 is used to calculate the delay time of an excavator (a loading machine) $t_{b}^{\prime}$ caused by waiting for a vehicle ( $i$-th demand) to arrive:

$$
t_{b i}^{\prime}=t_{i-1}^{p a b}-t_{i}^{i j}
$$

Then, the initial and the final time of $i$-th demand handling is determined:

$$
\left.\begin{array}{l}
t_{i}^{p r}=t_{i}^{i j} \\
t_{i}^{p a b}=t_{i}^{i j}+t_{a p t i}
\end{array}\right\}
$$

and the total delay time of handling equipment (an excavator) waiting for a vehicle to arrive is:

$$
\sum_{l=1}^{i} t_{b l}^{\prime}=\sum_{l=1}^{i-1} t_{b l}^{\prime}+t_{b i}^{\prime}
$$

The iteration 6 is used to determine the total time losses in handling $i$-th demand.

The iteration 7 is used to form the return time of a loaded (unloaded) vehicle $t_{g r i}$ ( $i$-th demand) according to the specified distribution law.

The iteration 8 determines the moment of the demand formulation for $a$-th vehicle:

$$
T_{a}=t_{i}^{p a b}+t_{g r i} .
$$

The iteration 9 is used to determine the number of demands to be handled:

$$
n_{i}=n_{i-1}+1 \text {. }
$$

The iteration 10 is used to check the conditions at the final stage of modelling, i.e. $n_{i}$ and $n_{r}$ (a number of hauls). When $n_{i}<n_{r}$, it is possible to go to $t_{i+1}^{i j}$ (iteration 1) applying the modelling of $i$-st demand instead of the initial data, while for $T_{k}$ data, the former moment of the vehicle arrival at the system is substituted with the value $T_{a}$. If $n_{i}=n_{r}$, the optimal value of the criterion for a given number of vehicles $m$ is found.

Therefore, we can pass to the iteration 11 to determine the delay time of a vehicle (demand) waiting for handling by special equipment (excavator):

$$
\bar{t}_{b}=\frac{\sum_{l=1}^{n_{r}} t_{b l}}{n_{r}} .
$$

The iteration 12 is used to calculate the delay time of an excavator waiting for the vehicles to arrive:

$$
\overrightarrow{t_{b}}=\frac{\sum_{l=1}^{n_{r}} t_{b l}^{\prime}}{n_{r}} .
$$

The iteration 13 is the value of the efficiency function $\Delta S_{k}$ (30). Then, the calculations are repeated for the cases when the number of vehicles is equal to $m+1, m+2$. If in this case the optimality criterion $\Delta S_{k}$ decreases, the number of trucks corresponding to $\Delta S_{k_{\min }}$ is considered an optimal alternative. When the values of $\Delta S_{k}$ increase, the alternatives with the number of vehicles $m+1, m+2, \ldots$, are calculated until an optimal solution is found for $\Delta S_{k_{\min }}$.

The iteration 14 is used to calculate the excavator output per hour $D_{v a l}^{\prime}$, when time losses for technical causes and breaks are not taken into account:

$$
D_{v a l}^{\prime}=\frac{q \gamma_{s t}}{\bar{t}_{a p t}+\overrightarrow{t_{b}}}
$$

With the help of the iteration 15 average vehicle output per hour is calculated:

$$
D_{v a l}=\frac{D_{v a l}^{\prime}}{m} .
$$

The iteration 16 is used to calculate the cost of the output and transportation of 1 ton of cargo:

$S_{i \check{s} . t}=\frac{C_{v a l}^{\prime}}{D_{v a l}^{\prime}}+\frac{l_{p a k . v}}{q \gamma_{s t} \beta}\left(C_{k i n}+\frac{C_{p a s}}{v_{T}}\right)+\frac{C_{p a s}\left(\bar{t}_{b}+\bar{t}_{a p t}+\bar{t}_{p}\right)}{q \gamma_{s t}}$

here $D_{v a l}^{\prime}-$ cost of 1 hour of excavator work, $\mathrm{Lt}$; $C_{k i n}$ - variable costs of $1 \mathrm{~km}$ of vehicle run; $C_{\text {pas }}$ - cost of 1 hour of vehicle delay time; $\bar{t}_{p}$ - average vehicle delay time at intermediate points, min.

$$
C_{v a l}^{\prime}=\frac{C_{p r}^{\prime} \vec{t}_{b}^{\prime}+C_{d a r}^{\prime} \bar{t}_{a p t}}{\vec{t}_{b}+\bar{t}_{a p t}}
$$

here $C_{p r}^{\prime}, C_{d a r}^{\prime}-$ cost of 1 hour delay time of excavation, cond. units.

\section{A complex solution of the problems of transport facilities selection and stock management}

Taking into account the dependence of transportation cost on vehicle capacity and size, the models of stock management and transport facilities selection may be analysed from various perspectives.

The cost of freight transportation varies depending on particular conditions.

When the amount of a particular lot of goods $g$ exceeds vehicle capacity $\left(g>q \gamma_{s t}\right)$, the cost of transportation does not depend on the amount of goods in a lot, implying that, in this case, the delivery cost of 1 ton of cargo is constant:

$$
S_{T}^{(1)}(g)=a_{1} ; g>q \gamma_{s t} \text {. }
$$

If the lot size corresponds to the capacity of a vehicle chosen from a number of vehicles in a queue 
$\left(g_{d}=q \gamma_{s t}\right)$, the delivery cost of 1 ton of goods may be obtained from the formula expressing the cost of freight transportation on the delivery routes, i.e., when $\bar{l}_{(i-1)-i}=0$ and $q \gamma_{s t}=g_{d}\left(q \gamma_{s t}\right.$ is a coefficient expressing the use of vehicle capacity determining its work load). In this case the following function describes the relationship between the delivery cost of 1 ton of cargo and a lot of goods:

$$
S_{T}^{(2)}(g)=a_{2}+\frac{b_{2}}{g}+c_{2} g ; g=q \gamma_{s t} \text {. }
$$

The delivery cost of 1 ton of cargo on the routes $g<q \gamma_{s t}$ depending on the average lot size is obtained from the following formula:

$$
S_{T}^{(3)}(g)=a_{3}+\frac{b_{3}}{g} ; g<q \gamma_{s t} .
$$

The cost of cargo storage consists of storage cost, natural loss of weight and losses due to the deterioration of consumer goods and the cost of goods 'freezing'. When containers are used for cargo delivery, the cost of their removal from the whole cycle should be taken into account.

The cost of the storage of goods $x$ in a unit of time is as follows:

$$
S_{\text {saug }}(x)=x C_{\text {saug }}+C_{\text {tar }} g_{t} k_{i \check{s} . p r},
$$

here $C_{\text {saug }}, C_{\text {tar }}$ - the cost of storing 1 ton of cargo and containers per unit time; $g_{t}$ - mass of containers in one cargo delivery, $\mathrm{t} ; k_{i \check{s} . p r}=t_{t . i \check{s}} / t_{p r s}-$ coefficient of container removal periodicity $\left(t_{t . i \check{s}}\right.$ and $t_{p r s}-$ time intervals between taking out of containers and cargo delivery).

Since

$$
g=g_{t}+g_{p}=\left(1+\frac{1}{k_{t}}\right) g_{t},
$$

here $g_{p}$ is mass of goods in one delivery operation, t; $k_{t}=g_{t} / g_{p}$ - container coefficient, then:

$$
S_{\text {saug }}(x)=x C_{\text {saug }}+\frac{C_{\text {tar }} k_{i \check{s} . p r} k_{t}}{1+k_{t}} g .
$$

The above model of managing stocks of goods when the cost of handling does not depend on the size of lots is simple. However, the following case describing the cost of handling a lot of goods $g$ is more common:

$$
S_{3}(g)=a_{u \check{z} . \dot{ } \cdot v}+b_{u z ̌ . i v} g
$$

here $a_{u \check{z} . \dot{ }, j}$ and $b_{u z ̌ . j v}$ are constant values.

The given above relationships, a model of the cost may be expressed as follows:

The cost of making an order for a lot of goods is:

$$
S_{3}(g)=\left\{\begin{array}{l}
a_{u \check{.} . \dot{v} v}+b_{u \check{.} . \dot{v}} g, g>0 ; \\
0, g=0
\end{array}\right.
$$

The cost of delivering 1 ton of cargo for a lot of goods $g$ is:

$$
S_{T}(g)=\left\{\begin{array}{l}
a_{1}, g>q \gamma_{s t} ; \\
a_{2}+\frac{b_{2}}{g}+c_{2} g, g=q \gamma_{s t} \\
a_{3}+\frac{b_{3}}{g}, g<q \gamma_{s t} .
\end{array}\right.
$$

The cost of stock storage $x$ per unit time is as follows:

$$
S_{\text {saug }}(x)=\left\{\begin{array}{l}
C_{\text {saug }} x+p g, x \geq 0 ; \\
p g, x<0 .
\end{array}\right.
$$

Let us consider the simplest management model of storing the same goods for a given constant demand with the intensity $r$. The delivery value $g$ connected with the relationship $g_{p}=g /\left(1+k_{t}\right)$ of the delivered lot of goods is unknown. When the demand and supply are known, there is no need for piling up goods, because a new order is made when the stock of goods is over. The function $x(t)=g_{p}-r t$ indicates the available stock of goods in the time interval $t$.

The dynamic problem is solved individually by considering a sequence of statistical problems for any interval of delivery $t_{p r s}=g_{p} / r$ specified by the conditions $x\left(t_{p r s}\right)=0$. An average stock value during a particular period of time is as follows:

$$
\bar{x}=\frac{1}{t_{p r s}} \int_{0}^{t_{p r s}} x(t) d t=\frac{g_{p}}{2}=\frac{g}{2\left(1+k_{t}\right)} .
$$

The relationship of the total cost calculated for one ton of cargo depending on the lot size is expressed in the following way:

$$
S(g)=\frac{S_{3}(g)}{g}+S_{T}(g)+\frac{S_{\text {saug }}(\bar{x}) t_{\text {prs }}}{g} .
$$

The considered alternatives of cargo delivery vary in the delivery cost $S_{T}(g)$. However, in any case, the relationship between the total cost and the size of cargo lot is described by the same formula:

$$
S(g)=a+\frac{b}{g}+c g,
$$

here only the coefficients a, b, c (Table) vary.

Based on the expression $\partial S(g) \partial g$ we can find the optimal lot size: 
Constant coefficients for determining the total cost by the formula (71)

\begin{tabular}{|c|c|c|c|}
\hline \multirow{2}{*}{ Delivery variant } & \multicolumn{3}{|c|}{ Coefficient } \\
\hline & $\mathrm{a}$ & $\mathrm{b}$ & $\mathrm{c}$ \\
\hline$g>q \gamma_{s t}$ & $a_{1}+a_{u \check{z} . \dot{v} v}$ & $a_{u z ̌ . i v}$ & $\frac{0,5 C_{\text {saug }}+C_{\text {tar }} k_{i \check{s} . p r} k_{t}}{\left(1+k_{t}\right)^{2} r}$ \\
\hline$g=q \gamma_{s t}$ & $a_{2}+b_{u \check{z} . \dot{i} v}$ & $a_{u \check{z} . \dot{. j v}}+b_{2}$ & $\frac{0,5 C_{\text {saug }}+C_{t a r} k_{i \check{s} . p r} k_{t}}{\left(1+k_{t}\right)^{2} r}+c_{2}$ \\
\hline$g<q \gamma_{s t}$ & $a_{3}+b_{u \check{z} . \dot{i v}}$ & $a_{u \check{z} . \dot{ } \cdot v}+b_{3}$ & $\frac{0,5 C_{\text {saug }}+C_{t a r} k_{i \check{s} . p r} k_{t}}{\left(1+k_{t}\right)^{2} r}$ \\
\hline
\end{tabular}

$g_{\text {opt }}=\sqrt{\frac{b}{c}}$.

The most optimal periodicity of load delivery is as follows:

$$
t_{\text {opt.prs }}=\frac{g_{p . o p t}}{r}=\frac{1}{\left(1+k_{t}\right) r} \sqrt{\frac{b}{c}} .
$$

Let us assume that in the first delivery option $\left(g>q \gamma_{s t}\right)$ the cargo is not delivered in containers, i.e. $k_{t}=0$, then we will obtain previously discussed Wilson's formula:

$$
g_{\text {opt }}=\sqrt{\frac{2 C_{u \check{z} r}}{C_{\text {saug }}}},
$$

here $C_{u \check{z}}$ - the observance of delivery terms. In the first model only the cost of delivery and storage is taken into account. The delivery of small lots of goods in short intervals is more expensive from the organizational point of view, while the delivery of big lots in long intervals increases storage cost.

The third model $\left(g<q \gamma_{s t}\right)$ takes into account the cost of delivery depending on the lot size, however, only on the routes where cargo is taken out. In this case the formula (72) determines the optimal average size of the cargo lot for several customers on the same route, while the lot size for individual customers should be determined depending on demand.

The coefficient $c$ depends on the same factors as those relating to the first model, while the value of coefficient $b$ is higher than that of $b_{3}$ depending on the average distance run by a vehicle between the two intermediate closest points $\bar{l}_{(i-1)-i}$, zero vehicle's run $l_{n}$, and additional delay time of a vehicle at every intermediate point $t_{3}$.

Since choosing the proper vehicle capacity for freight delivery on the dispatching routes is an extreme problem and the optimal vehicle capacity depends on the average lot size, the two problems including the choice of the lot size and vehicle capacity are solved together.
The average lot size and vehicle capacity are found from the following system of equalities:

$$
\left.\begin{array}{l}
\frac{\partial S\left(g_{d}, q \gamma_{s t}\right)}{\partial g_{d}}=0 ; \\
\frac{\partial S\left(g_{d}, q \gamma_{s t}\right)}{\partial\left(q \gamma_{s t}\right)}=0 ;
\end{array}\right\} g_{d}=g .
$$

The relationship between the total cost for 1 ton of cargo and the average lot size $g$ is expressed by the equality (71) obtained by substituting the function $S_{T}^{(3)}$ taken from the formula (61) into the formula (70).

By differentiating the equality expressing the total cost of transporting 1 ton of cargo according to $g_{d}$ and $q \gamma_{i \check{s}}$ and assuming the results to be equal to zero, we obtain the systems (76) and (77). One of them is as follows:

$$
g_{d}=\left(1+k_{t}\right) \times
$$

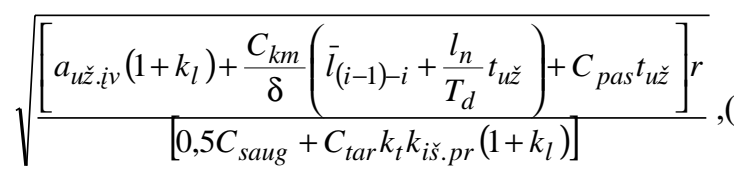

here $C_{k m}$ - cost of $1 \mathrm{~km}$ run of a vehicle, $k_{l}-$ coefficient taking into account additionally taken cargo, $T_{d}$ - time of vehicle operation, $t_{t}$ - time of unloading a unit cargo mass.

The second formula is expressed in the following way:

$$
q \gamma_{i \check{s}}=\sqrt{\frac{g_{d}\left(2 \bar{l}_{i}-\bar{l}_{(i-1)-i}\right) a_{k m}}{\left.b_{k m} \bar{l}_{(i-1)-i}+\left(\frac{l_{n}}{T_{d}} b_{k m}+\delta b_{p a s}\right) t_{t}\left(1+k_{l}\right) g_{d}+t_{u \check{z}}\right]}} \cdot
$$

The values $C_{k m}$ and $C_{p a s}$ in the above formula depend on vehicle capacity. The expression (77) determines the optimal capacity of vehicles operating on the routes for taking the cargo out. The system of the above equalities may be solved in a simple way by 
determining vehicle capacity and choosing an appropriate size of a lot of goods. Then, an optimal capacity may be determined for the vehicle. The calculations are made until the same results are obtained for two consecutive calculations, i.e. they yield the same value of vehicle capacity. The latter and the corresponding average size of a lot of goods will be considered optimal.

\section{Conclusions}

1. The investigation has shown that there is a linear relationship between the vehicle delay time and the number of arrivals at intermediate points, implying that the higher the number of arrivals, the longer the delay time.

2. The problem of transportation organization is considered in terms of mass service theory by minimizing unit cost of transportation.

3. The problems of stock management and proper choice of transport facilities are defined and analysed taking into account the relationship between cost of transportation and vehicle capacity and size of cargo lots.

By using mathematical statistical methods, the optimal vehicle capacity for a particular lot on the routes for taking the cargo out as well as the periodicity of cargo delivery are determined.

\section{References}

1. Nuzzolo, A.; Russo, F.; Crisali, U. A Doubly Dynamic Schedule - based Assignment Model for Transit Network. Transportation Science, Vol 35, No 3. August, 2001, p. 268-285.

2. Bodin, L.; Mingozzi, A.; Baldacci, R.; Ball, M. The Roll on - Roll off Vehicle Routing Problem. Transportation Science, Vol 34, No 3, August, 2000, p. 256-271.

3. Zhan, F.-B.; Noon, Ch.-F. Shortest Path Algorithms: An Evaluation using Real Road Networks. Transportation Science, Vol 32, No 1. February, 1998, p. 65-73.

4. Guelat, J.; Florion, M.; Crainic, T.-G. A Multimode Multiproduct Network Assignment Model for Strategic Planning of Freight Flows. Transportation Science, Vol 24, No 1. February, 1990, p. 25-39.

5. Baublys, A. Simulation of loadings on the railway terminal. Transport engineering (Transportas), Vol XV, No 3, Vilnius: Technika, 2000, p. 105-107 (in Lithuanian). 